\title{
Relación entre la actividad física, el sedentarismo y la calidad seminal
}

\author{
Paula Cristina Lalinde Acevedo ${ }^{1}$, José Manuel Mayorga Torres ${ }^{1}$, Walter D. Cardona Maya. ${ }^{1}$ \\ ${ }^{1}$ Grupo Reproducción, Facultad de Medicina, Universidad de Antioquia. Medellín, Colombia.
}

\section{RESUMEN}

La preocupación por el declive de los parámetros seminales humanos se ha incrementado en los últimos años, en especial por el aumento en las consultas por infertilidad. La calidad seminal puede verse influenciada por factores ambientales o propios del estilo de vida y dentro de los últimos se encuentra la práctica de actividad física. Los resultados de los estudios que han evaluado los efectos del estilo de vida físicamente activo sobre la calidad seminal han sido contradictorios, por lo que la relación entre ambos no está claramente establecida; no obstante, se ha propuesto que la práctica de actividad física podría tener efectos positivos sobre la calidad seminal, así como tiene diferentes beneficios para la salud. La posibilidad de incluir la mejoría en la calidad seminal dentro de las razones para incentivar el estilo de vida físicamente activo en las personas, necesita del aumento en el conocimiento acerca de la influencia que tienen diversos aspectos involucrados en la práctica de actividad física, sobre los parámetros espermáticos. El objetivo de esta revisión de la literatura es recopilar los principales aspectos relacionados con el efecto del estilo de vida, en especial de la actividad física y el sedentarismo, sobre la calidad seminal.

\section{PALABRAS CLAVE: Calidad seminal, estilo de vida, actividad física, sedentarismo}

\section{SUMMARY}

Concern about the decline in human semen parameters has grown in recent years, due in part to the increase in infertility consultations. Semen quality may be influenced by environmental or lifestyle factors such as the practice of physical activity but the relation between semen quality and physically active lifestyle is not clearly established. Nevertheless, it has been proposed that physical activity might have positive effects on semen quality as well as it has various health benefits. To possibly include the improvement of semen quality on the reasons to stimulate the physically active lifestyle in people, it's necessary to increase the knowledge about the influence of diverse aspects involved on the practice of physical activity on the sperm parameters. The purpose of this literature review is to compile the main aspects related to the effect of lifestyle, particularly physical activity and sedentarism on semen quality.

KEY WORDS: Semen quality, lifestyle, physical activity, sedentarism 


\section{INTRODUCCIÓN}

En los últimos 25 años se ha incrementado la preocupación por la calidad seminal humana debido a la evidencia que demuestra el declive de los parámetros seminales convencionales en los últimos 60 años $(1,2)$, lo que a su vez se ha relacionado con los problemas de fertilidad que atraviesan alrededor del $15 \%$ de las parejas en edad reproductiva en el mundo (3-5), en los cuales, el factor masculino está involucrado como la causa principal o en combinación con una alteración femenina en al menos el $50 \%$ de los casos (6).

Carlsen y cols en 1992, evidenciaron por primera vez la reducción progresiva y significativa de la concentración espermática y el volumen seminal, mediante la recopilación de información desde 1935 hasta 1991, en un estudio retrospectivo de 61 trabajos científicos (2). Estos resultados fueron apoyados posteriormente por otros investigadores $(7,8)$, sin embargo, ha sido un trabajo ampliamente criticado debido a que no tiene en cuenta la heterogeneidad entre los estudios analizados en cuanto a las variaciones en la calidad seminal por regiones geográficas, por individuo y por eyaculado, así como en la metodología empleada para analizar la información $(1,9)$.

La calidad espermática puede verse influenciada por factores de tipo ambiental o propios del estilo de vida de la persona, los cuales se han considerado como los causantes de algunas alteraciones testiculares y problemas reproductivos $(10,11)$, afectando el potencial fértil del hombre y desencadenando efectos nocivos tanto en el adulto, como en el feto en formación $(12,13)$.

Entre los factores ambientales se han incluido la exposición prolongada y continua: i) al calor y al aumento de la temperatura del escroto $(9,14)$; ii) a contaminantes ambientales con potencial efecto disruptor del sistema endocrino $(9,15)$ y iii) a radiaciones electromagnéticas (11). Entre los factores relacionados con el estilo de vida se encuentran, el fumar, el uso recreativo de drogas, la ingesta de alcohol, la dieta, la obesidad y el sedentarismo $(9,16,17)$.

No se puede desconocer que en los últimos 50 años se han generado cambios importantes en muchos aspectos que involucran el estilo de vida de la población humana, en especial en lo que se refiere a la actividad física (16). El desarrollo acelerado de diferentes tecnologías que facilitan las actividades cotidianas o que son diseñadas para el esparcimiento, la diferencia entre los empleos y los pasatiempos antiguos respecto a los actuales que involucran la prevalencia de posiciones sedentarias
$(18,19)$, así como los avances en los sistemas de transporte y la conectividad dentro y entre las ciudades $(15,20)$, son aspectos que pueden repercutir en la promoción y consecuente incremento de conductas sedentarias.

Dentro de los beneficios de la actividad física sobre la salud humana están, la reducción del riesgo de padecer cardiopatías coronarias, accidentes cerebrovasculares, diabetes tipo II, obesidad, depresión, cáncer de colon y de mama, además de prevenir la pérdida de masa muscular y de densidad ósea $(21,22)$. Adicionalmente, la actividad física puede tener efectos positivos sobre la calidad espermática. Varios autores han evaluado la relación entre la calidad seminal y la práctica de actividad física (22-29) con resultados contradictorios, lo que contribuye a que aún no esté bien establecida la relación entre ambas $(9,18,22)$.

El objetivo de esta revisión de la literatura es recopilar los principales aspectos relacionados con el efecto del estilo de vida, en especial de la actividad física y el sedentarismo, sobre la calidad seminal.

\section{La calidad seminal}

La calidad seminal se ha evaluado tradicionalmente mediante el análisis seminal o espermograma, en el cual se determinan un conjunto de parámetros como el volumen del eyaculado, la movilidad, la concentración, la morfología y la viabilidad espermática siguiendo los lineamientos establecidos por la Organización Mundial de la Salud en el manual de laboratorio para el examen y procesamiento de semen humano (30). No obstante, este análisis convencional continúa siendo subjetivo y no permite evaluar diferentes propiedades funcionales de los espermatozoides $(31,32)$, ni detectar anormalidades fisiológicas en las células espermáticas que pueden contribuir al incremento de las estadísticas de infertilidad (5).

En el caso particular del daño al ADN espermático, éste puede ser causado por diferentes mecanismos, entre ellos se incluyen factores intrínsecos tales como anormalidades en la espermatogénesis de origen genético o que ocurren durante el desarrollo gamético y un remodelamiento insuficiente de la cromatina (33), también por estresores endógenos y exógenos $(33,34)$ como las especies reactivas del oxígeno (ERO), las cuales podrían estar presentes en el ambiente testicular (33).

Aunque una generación limitada de ERO es crítica para desencadenar las señales celulares que faciliten los procesos de hiperactivación, capacitación, reacción acrosomal y unión del espermatozoide a la zona pelúcida del oocito $(5,35)$, un exceso 
de éstas puede impactar negativamente la calidad espermática, al interferir con la fluidez de la membrana celular, provocando fallos en la capacidad de interacción entre el espermatozoide y el oocito (5), además de afectar componentes estructurales del axonema, repercutiendo en la movilidad de los espermatozoides (36).

Adicionalmente, la producción de excesivas cantidades de ERO en eventos de estrés oxidativo, puede causar fragmentación en las cadenas de ADN $(37,38)$, afectar el proceso de condensación de la cromatina $(5,35,38)$ y ser un factor limitante para la reproducción, debido a que se ha observado que afecta el desarrollo temprano del embrión (39) y se ha relacionado con la pérdida recurrente del embarazo temprano $(13,37,40)$ y con la reducción del potencial fértil masculino $(4,32,33)$.

Por mucho tiempo la concentración y la movilidad espermática fueron considerados los principales indicadores del potencial fértil del hombre (1), sin embargo, otras características espermáticas como la morfología, toman relevancia al tener en cuenta que los espermatozoides anormales exhiben un exceso de citoplasma como resultado de un proceso espermatogénico alterado (35) y producen una mayor cantidad de ERO $(27,32,36,37)$.

A raíz de todo lo anterior, técnicas adicionales al análisis seminal convencional, las cuales permiten la determinación de la cantidad de ERO producidas por los espermatozoides $(5,35)$, evaluar el potencial de membrana mitocondrial (32), el índice de fragmentación de la cromatina espermática $(32,37,41)$ y la lipoperoxidación de la membrana espermática $(32,42)$, se han desarrollado en las últimas décadas como complemento a la determinación de la calidad seminal (43).

\section{Relación entre la actividad física y la calidad seminal}

La actividad física es definida como cualquier movimiento corporal voluntario, repetitivo, que involucre los grandes grupos musculares (21) y aumente sustancialmente el gasto energético por encima del estado de reposo $(20,44)$. Puede comprender las actividades cotidianas o adoptar diversas formas tales como aeróbica, de fortalecimiento, de flexibilidad o de equilibrio, por lo que engloba al ejercicio y el deporte $(21,45)$.

Se cataloga de acuerdo a la intensidad con la que sea practicada, como actividad física modera$\mathrm{da}$, que acelera de forma perceptible el ritmo cardiaco e incluye caminar, hacer yoga, estiramiento, bajar escaleras, hacer trabajos de jardinería leves, ejecutar las actividades domésticas, pasear en bicicleta, nadar recreativamente, entre otras $(21,46)$, o como actividad física vigorosa la cual requiere un esfuerzo físico mayor, provoca una respiración rápida y un aumento sustancial en la frecuencia cardiaca; incluye correr, caminar a paso rápido o en ascenso, escalar, bailar a un ritmo intenso, desplazamientos rápidos en bicicleta, saltar la cuerda, practicar aeróbicos, deportes y juegos competitivos, el entrenamiento con rutina de ejercicios en el gimnasio, el trabajo cardiovascular, entre otros $(21,46)$.

En los estudios realizados en atletas de triatlón (26) y en ciclistas profesionales (25), así como en corredores con entrenamiento moderado (54-72 $\mathrm{Km} /$ semana) (23), no se observaron alteraciones en la calidad seminal al compararlos con hombres sedentarios, a excepción de la reducción en la movilidad espermática manifestada en el grupo de ciclistas, únicamente en el periodo de competencia (25). Esto contrasta con lo observado en deportistas de alto rendimiento, tales como corredores de maratón con un entrenamiento exigente de aproximadamente $100 \mathrm{Km} / \mathrm{semana}$, en los que la movilidad espermática estaba reducida (24) y el número de células inmaduras en el eyaculado estaba aumentado (26).

Al evaluar los parámetros seminales convencionales de jugadores de polo acuático y atletas de triatlón, Vaamonde y cols en 2009, obtuvieron resultados similares en los cuales se manifiestan valores reducidos de los parámetros espermáticos, inversamente proporcionales al volumen e intensidad de la actividad física practicada, además, observaron una marcada alteración en la morfología espermática respecto a los valores encontrados en hombres que practican actividad física pero no de alto rendimiento (27).

Por otro lado, Wise y cols en 2011, no observaron asociación entre la actividad física regular, tanto moderada como vigorosa y los parámetros seminales, a excepción del subgrupo de individuos que montaban bicicleta regularmente, en los que la concentración espermática disminuyó (29). Por el contrario, Campos y cols (2011) observaron valores significativamente aumentados para el volumen del eyaculado, la concentración, la movilidad espermática y el porcentaje de normozoospermia en hombres clasificados como físicamente activos habitualmente respecto a los físicamente activos ocasionales y los sedentarios que acudieron a un centro de fertilidad. Además al agruparlos de acuerdo al índice de masa corporal (IMC), el recuento de espermatozoides móviles y de normozoospermia 
tuvo un descenso significativo en los hombres con IMC mayor de $25 \mathrm{Kg} / \mathrm{m} 2$, quienes en su mayoría estaban en el grupo de hombres sedentarios (28).

De igual modo, Vaamonde y cols en 2012, observaron valores significativamente más altos para la movilidad progresiva total y la morfología espermática de hombres que practicaron por más de un año continuamente algún tipo de actividad física vigorosa, excepto montar en bicicleta, frente a hombres sedentarios (22). Resultados similares fueron encontrados por Jurewicz y cols en 2014, quienes al evaluar diferentes componentes del estilo de vida y su efecto en la calidad seminal, observaron que la práctica recreativa constante de actividad física tanto moderada como vigorosa estaba relacionada positivamente con la concentración espermática (17).

\section{Relación entre el sedentarismo y la calidad seminal}

El sedentarismo es una conducta caracterizada por el gasto de menos del $10 \%$ de la energía diaria en realizar actividades de moderada o alta intensidad (47). Se asocia a un conjunto de actividades pasivas practicadas por la persona continuamente o durante la mayor parte del día como: el permanecer recostado, ver televisión, dormir, el uso del computador y la alta propensión a estar sentado por un tiempo prolongado (48). No hacer ejercicio o no practicar algún deporte regularmente son actitudes que se incluyen dentro de lo que define a una persona sedentaria $(48,49)$.

El estudio del sedentarismo y su relación con la calidad espermática se ha asociado con la relación entre la fertilidad masculina y la obesidad, debido a que ésta es una de las enfermedades con más impacto en la población humana por todos los problemas secundarios de salud que acarrea $(50,51)$ y el incremento acelerado del número de casos en el mundo $(52,53)$; además, es un importante factor del estilo de vida que puede afectar adversamente la espermatogénesis (28).

Chavarro y cols en 2010, no encontraron diferencias en la concentración, la movilidad y la morfología espermática en relación al incremento en el IMC, sin embargo, observaron que el número total de espermatozoides por eyaculado fue menor en los hombres con IMC $\geq 35 \mathrm{Kg} / \mathrm{m} 2$, los cuales a su vez, tenían mayor cantidad de espermatozoides con daño en el ADN frente a los hombres con IMC normal, además el volumen del eyaculado fue menor a medida que aumentaba el IMC (51). Esto concuerda con lo observado por otros autores $(17,50)$, pero difiriere de los resultados del estudio de Kort y cols en 2006, en que el IMC estuvo inversamente relacionado con la cantidad de espermatozoides normales y móviles (54). Eisenberg y cols en 2013, observaron además, que el número total de espermatozoides por eyaculado tuvo una asociación negativa con la circunferencia de la cintura pero no hubo diferencias significativas en los niveles de fragmentación de la cromatina (50).

Se considera que los individuos obesos tienden a tener un estilo de vida más sedentario y el IMC elevado repercute en un aumento en la deposición de grasa en las caras internas de los muslos y la región suprapúbica, lo que puede influenciar el intercambio normal de calor en los testículos $(52,54)$. La temperatura es un aspecto fundamental en el desarrollo de los gametos masculinos de los mamíferos y la exposición testicular a una mayor temperatura directamente y por un tiempo prolongado puede afectar la espermatogénesis $(9,55,56)$, debido a la mayor sensibilidad de las membranas mitocondriales de las células germinales al estrés por calor (56), y resultar en elevados niveles de apoptosis, autofagia, necrosis y detención del ciclo celular (57). El calor está relacionado con el desarrollo y fisiopatología de algunos desordenes reproductivos como varicocele (58), además, puede inducir en los testículos estrés oxidativo producido por las ERO (38) y desencadenar apoptosis espermática $(56,57,59)$.

Se asume generalmente que la temperatura testicular un poco más baja que la abdominal, aproximadamente $34^{\circ} \mathrm{C}$, reduce las tasas de daño oxidativo en el ADN espermático $(14,57)$ y de ahí la explicación a la hipótesis evolutiva de la exteriorización de los testículos en el escroto, característica única de los mamíferos, cuyo argumento es que permite proveer el ambiente local más adecuado para la producción, maduración y almacenamiento de las células espermáticas $(14,60)$.

\section{El sedentarismo en el lugar de trabajo}

El comportamiento sedentario se ha asociado con el incremento en la temperatura testicular al mantener ciertas posturas corporales durante un tiempo prolongado $(12,55,60)$. Hjollund y cols en 2000 , evidenciaron una mayor prevalencia de baja concentración espermática en hombres con posturas ocupacionales sedentarias en comparación con hombres que están la mayor parte de la jornada laboral con posturas no sedentarias y tienen un trabajo activo (55). Conclusiones similares fueron obtenidas por Magnusdottir y cols en 2005, al evaluar 
individuos de profesiones como conducir un vehículo la mayor parte del día, la cual se ha relacionado con el incremento de la temperatura testicular, la manifestación de fertilidad reducida y más baja producción espermática (15), además, este tipo de empleos favorecen el aumento de la temperatura del escroto en $0,7^{\circ} \mathrm{C}(55)$. En contraste Stoy y cols en 2004, no encontraron diferencias significativas en la concentración espermática, en hombres con altas proporciones de trabajo sedentario, sentados la mayor parte de la jornada, en comparación con los que tienen bajas proporciones de éste (19).

\section{DISCUSIÓN}

La inactividad física es reconocida como el cuarto factor de riesgo de mortalidad en el mundo y se ha catalogado como uno de los principales problemas de salud pública del presente siglo $(52,61)$, principalmente por su relación con las cardiopatías coronarias, los accidentes cerebrovasculares, el desarrollo de obesidad, la diabetes tipo II, la osteoporosis e incluso con la manifestación de algunos tipos de cáncer $(21,44)$. A raíz de esto, la OMS ha planteado varias recomendaciones para que en los países se formulen políticas y estrategias que promuevan el incremento de la actividad física de sus habitantes, como la de incentivar a la población a realizar por lo menos 30 minutos de actividad física mínimo tres veces por semana de forma recreacional $(21,45)$.

El sedentarismo y el aumento del tamaño corporal (IMC o la circunferencia de cintura) se han visto relacionados negativamente con los parámetros seminales, posiblemente por su relación con el incremento de temperatura en los testículos y el efecto del calor en las células espermatogénicas en desarrollo $(17,28,50,51)$. Como la infertilidad afecta aproximadamente al $15 \%$ de las parejas en el mundo con impactos significativos en su calidad de vida, la identificación y modificación de ciertos factores de riesgo potenciales como la relación entre la actividad o inactividad física y la calidad seminal, puede permitir a algunos individuos alcanzar sus metas reproductivas (50). Esto no significa necesariamente que la práctica de actividad física vigorosa o un entrenamiento elevado son la única solución, debido a que aunque lo presentado en esta revisión incluye generalmente a deportistas, la actividad física moderada pero constante tiene buenas consecuencias para el perfil hormonal (62) y para el estado de ánimo y corporal en general (21), por lo que puede repercutir positivamente en el potencial fértil del hombre.
La alta variabilidad en los parámetros seminales le resta poder a los estudios de calidad seminal, además la forma de analizar los datos, el número de hombres examinados y la heterogeneidad en las muestras seminales de acuerdo a la población, hacen difícil la comparación entre estudios (17). Sin embargo, las diferentes pruebas funcionales toman importancia como una herramienta más objetiva de evaluación de la calidad seminal, cuando se tiene en cuenta que son muy útiles en el diagnóstico de casos de infertilidad idiopática $(5,32)$ cuyas causas no son evidentes mediante el análisis seminal convencional $(6,31)$, además podrían ser mejores predictores del potencial fértil de un individuo que los parámetros seminales convencionales como lo demuestra el uso de pruebas para detectar la integridad de la cromatina y del ADN espermático en centros de reproducción asistida $(33,39)$. De igual modo, el implementar diferentes pruebas funcionales complementarias al análisis seminal convencional puede contribuir a un mejor diagnóstico de los problemas de fertilidad del hombre, para definir con mayor precisión un tratamiento y que en éste posiblemente se incluyan la mejoría de ciertos aspectos relacionados con el estilo de vida como lo es la práctica de actividad física.

\section{CONCLUSIONES}

Dentro de las políticas gubernamentales son necesarias diferentes formas de incentivar a las personas a practicar actividad física con regularidad, dada la evidencia de los diferentes beneficios para la salud corporal y mental que de su práctica se derivan y la reducción potencial de los costos que conllevan los tratamientos de enfermedades no transmisibles como la obesidad, para los sistemas de salud $(21,44)$. Sería interesante considerar la posibilidad de incluir la mejoría en la calidad seminal dentro de estos propósitos, pero esto sólo puede ser posible si se incrementa el conocimiento que permita clarificar los diversos factores que intervienen en la relación del estilo de vida físicamente activo o el sedentario con la calidad espermática y aspectos como la frecuencia, intensidad y duración de la práctica de actividad física que pueden repercutir directamente sobre la calidad seminal o también a través de su efecto en la regulación hormonal de los procesos espermatogénicos.

AGRADECIMIENTOS: Al Centro de Investigaciones en Ciencias Exactas y Naturales CIEN, (Proyecto código TE20110) y a la Estrategia de Sostenibilidad 2013/2014 del Grupo Reproducción de la Universidad de Antioquia, por el apoyo económico. 


\section{REFERENCIAS}

1. Merzenich $\mathrm{H}$, Zeeb $\mathrm{H}$, Blettner $\mathrm{M}$. Decreasing sperm quality: a global problem? BMC Public Health 2010;10:24

2. Carlsen E, Giwercman A, Keiding N, Skakkebaek NE. Evidence for decreasing quality of semen during past 50 years. BMJ 1992;305(6854):609-13.

3. Dohle GR, Colpi G, Hargreave T, Papp G, Jungwirth A, Weidner W. EAU guidelines on male infertility. Eur Urol 2005;48(5):703-11.

4. Bungum M. Sperm DNA integrity assessment: a new tool in diagnosis and treatment of fertility. Obstet Gynecol Int 2012;2012:531042.

5. Henkel R. ROS and semen quality. En: Agarwal A, Aitken RJ, Alvarez JG. editores. Studies on men's health and fertility. Oxidative Stress in Applied Basic Research and Clinical Practice. 1 ed. New York (U.S.A.): Humana Press, Springer; 2012. Pp. 301-23.

6. Meacham RB. Non Surgical Therapy. En: Niederberger $\mathrm{C}$, editor. An introduction to male reproductive medicine. 1 ed. United States: Cambridge University Press; 2011. Pp. 77-84

7. Auger J, Kunstmann JM, Czyglik F, Jouannet P. Decline in semen quality among fertile men in Paris during the past 20 years. N Eng J Med 1995;332(5):281-5.

8. Irvine DS. Declining sperm quality: a review of facts and hypotheses. Bailliere's Clin Obstet Gynecol 1997;11(4):655-71.

9. Sharpe RM. Environment, lifestyle and male infertility. Baillieres Best Pract Res Clin Endocrinol Metab 2000;14(3):489-503.

10. Agarwal A, Desai NR, Ruffoli R, Carpi A. Lifestyle and testicular dysfunction: a brief update. Biomed Pharmacother 2008;62(8):550-3.

11. Mendiola J, Torres-Cantero AM, Agarwal A. Lifestyle factors and male infertility: an evidence-based review. Arch Med Sci 2009;5(1A):S3-12.

12. Bonde JP, Storgaard L. How work-place conditions, environmental toxicants and lifestyle affect male reproductive function. Int J Androl 2002;25(5):262-8.

13. Vasco GC, Gil Villa AM, Piedrahita Ochoa C, Cardona Maya W, Cadavid Jaramillo A. Influencia de la impronta genómica masculina en la reproducción. Actas Urol Esp 2008;32(10):1004-12.

14. Ivell R. Lifestyle impact and the biology of the human scrotum. Reprod Biol Endocrinol 2007;5:15.

15. Magnusdottir EV, Thorsteinsson T, Thorsteinsdottir S, Heimisdottir M, Olafsdottir K. Persistent organochlorines, sedentary occupation, obesity and human male subfertility. Hum Reprod 2005;20(1):208-15.

16. Kumar S, Kumari A, Murarka S. Lifestyle factors in deteriorating male reproductive health. Indian J Exp Biol 2009;47(8):615-24.

17. Jurewicz J, Radwan M, Sobala W, Ligocka D, Radwan $P$, Bochenek $M$, et al. Lifestyle and semen quality: role of modifiable risk factors. Syst Biol Reprod Med 2014;60(1):43-51.

18. Sharpe RM. Lifestyle and environmental contribution to male infertility. Br Med Bull 2000;56(3):630-42.

19. Stoy J, Hjollund NH, Mortensen JT, Burr H, Bonde JP.
Semen quality and sedentary work position. Internat $\mathrm{J}$ Androl 2004;27(1):5-11.

20. Brownson RC, Boehmer TK, Luke DA. Declining rates of physical activity in the United States: what are the contributors? Annual Rev Public Health 2005;26:42143.

21. Organización Mundial de la Salud. Recomendaciones mundiales sobre actividad física para la salud. Ginebra (Suiza): Ediciones de la OMS; 2010. Disponible en: www.who.int/dietphysicalactivity/factsheet_recommendations/es/.

22. Vaamonde D, Da Silva-Grigoletto ME, Garcia-Manso JM, Barrera N, Vaamonde-Lemos R. Physically active men show better semen parameters and hormone values than sedentary men. Eur $\mathrm{J}$ Appl physiol 2012;112(9):3267-73.

23. Bagatell CJ, Bremner WJ. Sperm counts and reproductive hormones in male marathoners and lean controls. Fertil Steril 1990;53(4):688-92.

24. Arce JC, De Souza MJ, Pescatello LS, Luciano AA. Subclinical alterations in hormone and semen profile in athletes. Fertil Steril 1993;59(2):398-404.

25. Lucía A, Chicharro JL, Pérez M, Serratosa L, Bandrés F, Legido JC. Reproductive function in male endurance athletes: sperm analysis and hormonal profile. J Appl Physiol 1996;81(6):2627-36.

26. De Souza M, Arce J, Pescatello L, Scherzer H, Luciano A. Gonadal hormones and semen quality in male runners. A volume threshold effect of endurance training. Int J Sports Med 1994;15(7):383-91.

27. Vaamonde D, Da Silva-Grigoletto ME, Garcia-Manso JM, Vaamonde-Lemos R, Swanson RJ, Oehninger $\mathrm{SC}$. Response of semen parameters to three training modalities. Fertil Steril 2009;92(6):1941-6.

28. Campos M, Delgado E, Morgado S, Sanchez-Correa B, Gonzales MR, Gordillo J, et al. Influncia del ejercicio físico y el índice de masa corporal sobre la calidad espermática: análisis en pacientes de reproducción asistida. Revista de Embriología Clínica y Biología de la Reproducción ASEBIR 2011;16(1):25 - 32.

29. Wise LA, Cramer DW, Hornstein MD, Ashby RK, Missmer SA. Physical activity and semen quality among men attending an infertility clinic. Fertil Steril 2011;95(3):1025-30.

30. World Health Organization. Laboratory manual for the examination and processing of human semen 2010. Disponible en: http://www.who.int/reproductivehealth/ publications/infertility/9789241547789/en/.

31. Bungum M, Bungum L, Giwercman A. Sperm chromatin structure assay (SCSA): a tool in diagnosis and treatment of infertility. Asian J Androl 2011;13(1):6975.

32. Mayorga-Torres BJ, Cardona-Maya W, Cadavid A, Camargo M. [Evaluation of sperm functional parameters in normozoospermic infertile individuals]. Actas Urol Esp 2013;37(4):221-7.

33. Zini A, Albert O, Robaire B. Assessing sperm chromatin and DNA damage: clinical importance and development of standards. Andrology 2014;2(3):322-5.

34. Cordelli E, Eleuteri P, Leter G, Rescia M, Spano M. Flow cytometry applications in the evaluation of sperm 
quality: semen analysis, sperm function and DNA integrity. Contraception 2005;72(4):273-9.

35. Aitken RJ, Bennetts L. Reactive oxygen species: friend or foe. En: De Jonge JC, Barrat CLR, editores. The sperm cell: Production, Maturation, Fertilization, Regeneration. 1 ed. New York (U.S.A.): Cambridge University Press; 2006. pp. 170 - 93.

36. El-Taieb MA, Herwig R, Nada EA, Greilberger J, Marberger $M$. Oxidative stress and epididymal sperm transport, motility and morphological defects. Eur J Obstet Gynecol Reprod Biol 2009;144 Suppl 1:S199203.

37. Gil-Villa AM, Cardona-Maya W, Agarwal A, Sharma $\mathrm{R}$, Cadavid A. Role of male factor in early recurrent embryo loss: do antioxidants have any effect? Fertil Steril 2009;92(2):565-71.

38. Aitken RJ, Smith TB, Jobling MS, Baker MA, De luliis GN. Oxidative stress and male reproductive health. Asian J Androl 2014;16(1):31-8.

39. Cohen-Bacrie $P$, Belloc $S$, Ménézo YJ, Clement $P$, Hamidi J, Benkhalifa M. Correlation between DNA damage and sperm parameters: a prospective study of 1,633 patients. Fertil Steril 2009;91(5):1801-5.

40. Gil-Villa AM, Cardona-Maya W, Agarwal A, Sharma R, Cadavid A. Assessment of sperm factors possibly involved in early recurrent pregnancy loss. Fertil Steril 2010;94(4):1465-72.

41. Evenson D, Jost L. Sperm chromatin structure assay is useful for fertility assessment. Methods Cell Sci 2000;22(2-3):169-89.

42. Aitken RJ, Wingate JK, De luliis GN, McLaughlin EA. Analysis of lipid peroxidation in human spermatozoa using BODIPY C11. Molec Hum Reprod 2007;13(4): 203-11.

43. Cardona Maya WD, Berdugo Gutierrez JA, de los Rios J, Cadavid Jaramillo AP. Functional evaluation of sperm in Colombian fertile men. Arch Esp Urol 2007;60(7):827-31.

44. Duperly JS, Parra OL, Angarita-Gómez D, Rivera CL, Granados D, Donado C. Documento técnico con los contenidos de direccionamiento pedagógico para la promoción de hábitos de vida saludable, con énfasis en alimentación saludable y el fomento de ambientes $100 \%$ libres de humo de cigarrillo a través de la práctica regular de la actividad física cotidiana, dirigidos a los referentes de las entidades territoriales. Bogotá, Colombia. 2011. pp. 138.

45. Blair SN, LaMonte MJ, Nichaman MZ. The evolution of physical activity recommendations: how much is enough? American J Clin Nutr 2004;79(5):913S-20S.

46. Ainsworth BE, Haskell WL, Whitt MC, Irwin ML, Swartz AM, Strath SJ, et al. Compendium of physical activities: an update of activity codes and MET intensities. Med Sci Sports Exer 2000;32(9 Suppl):S498-504.

47. Bernstein MS, Morabia A, Sloutskis D. Definition and prevalence of sedentarism in an urban population. American J Public Health 1999;89(6):862-7.

48. Fox M. What is sedentarism. J Acad Nutr Diet 2012;112(8):1125-8.
49. Bauman AE, Reis RS, Sallis JF, Wells JC, Loos RJ, Martin BW, et al. Correlates of physical activity: why are some people physically active and others not? Lancet 2012;380(9838):258-71.

50. Eisenberg ML, Kim S, Chen Z, Sundaram R, Schisterman EF, Buck Louis GM. The relationship between male BMI and waist circumference on semen quality: data from the LIFE study. Hum Reprod 2013;29(2):193-200.

51. Chavarro JE, Toth TL, Wright DL, Meeker JD, Hauser R. Body mass index in relation to semen quality, sperm DNA integrity, and serum reproductive hormone levels among men attending an infertility clinic. Fertil Steril 2010;93(7):2222-31.

52. Tunc O, Bakos HW, Tremellen K. Impact of body mas index on seminal oxidative stress. Andrologia 2010;43:121-8.

53. Organización Mundial de la Salud. Cifras y datos: 10 datos sobre la obesidad 2013.Disponible en: http:// www.who.int/features/factfiles/obesity/es/. Acceso en: Febrero 20 de 2014.

54. Kort HI, Massey JB, Elsner CW, Mitchell-Leef D, Shapiro DB, Witt MA, et al. Impact of body mass index values on sperm quantity and quality. $J$ Androl 2006;27(3):450-2.

55. Hjollund NH, Bonde JP, Jensen TK, Olsen J. Diurnal scrotal skin temperature and semen quality. The Danish First Pregnancy Planner Study Team. Internat J Androl 2000;23(5):309-18.

56. Shiraishi K. Heat and oxidative stress in germ line. En: Agarwal A, Aitken RJ, Alvarez JG, editores. Studies on men's health and fertility. Oxidative Stress in Applied Basic Research and Clinical Practice. 1 ed. New York (U.S.A.). Humana Press, Springer; 2012. pp. 149-78.

57. Santiso R, Tamayo M, Gosalvez J, Johnston S, Marino A, Fernandez C, et al. DNA fragmentation dynamics allows the assessment of cryptic sperm damage in human: evaluation of exposure to ionizing radiation, hyperthermia, acidic $\mathrm{pH}$ and nitric oxide. Mutat Res 2012;734(1-2):41-9.

58. Zini A, Al-hathal N. Varicocele and oxidative stress. En: Agarwal A, Aitken RJ, Alvarez JG, editores. Studies on men's health and fertility. Oxidative Stress in Applied Basic Research and Clinical Practice. 1 ed. New York (U.S.A.). Humana Press, Springer; 2012. pp. 149-78.

59. Aitken RJ, Roman SD. Antioxidant systems and oxidative stress in the testes. Oxid Med Cell Longev 2008;1(1):15-24.

60. Koskelo R, Zaproudina N, Vuorikari K. High scrotal temperatures and chairs in the pathophysiology of poor semen quality. Pathophysiol 2005;11(4):221-4.

61. Blair SN. Physical inactivity: the biggest public health problem of the 21st century. Br J Sports Med 2009;43(1):1-2.

62. Lanfranco F, Minetto MA. The Male Reproductive System, Exercise, and Training: Endocrine Adaptations. Endocrinology of Physical Activity and Sport: Springer; 2013. pp. 121-32. 